\title{
Towards automatic non-metric traits analysis on 3D models of skulls
}

\author{
Bruno Andrade†, Paulo Dias†, Catarina Coelho*, João Coelho*, David Navega*, Sofia N. Wasterlainł, Maria Teresa \\ Ferreira*, Beatriz Sousa Santosं \\ $\dagger$ Departamento de Eletrónica, Telecomunicações e Informática, DETI / IEETA, Univ. Aveiro, Portugal \\ *Laboratório de Antropologia Forense, Centro de Ecologia Fun-cional, Departamento de Ciências da Vida, Univ. \\ Coimbra, Portugal \\ †Centro de Investigação em Antropologia e Saúde, Departamento de Ciências da Vida, Univ. Coim-bra, Portugal
} Email: brunoaandrade@ua.pt

\begin{abstract}
The morphological and metric methods used by anthropologists to assess ancestry can generate results with low repeatability besides damaging the specimens while handling. These problems have led to the development of a new approach based on skulls acquisition with a 3D scanner, using the resulting models to make measurements and morphological analyzes in the CraMs application (Craniometric Measurements). This paper focuses on the development of new methods for the morphological analysis, and the extraction and classification of structures with the objective of reducing inter and intra observer variability. The final aim is to ease the process of estimating the individual's ancestry.
\end{abstract}

Keywords - Skull Morphology; Ancestry; 3D Models; Feature Detection; Segmentation

\section{INTRODUÇÃO}

Tanto em Antropologia forense como na Antropologia das populações do passado recorre-se ao crânio para estimar quer a ancestralidade, quer o sexo, usando-se abordagens métricas e/ou morfológicas [1]. Porém, e apesar da contínua investigação nesta área, persistem alguns problemas difíceis de ultrapassar, a saber: 1) o contínuo manuseamento dos espécimes (questão particularmente pertinente no caso de exemplares arqueológicos), sobretudo durante a análise métrica (devido à utilização de instrumentos metálicos), que pode causar degradação óssea; 2) a baixa repetibilidade apresentada por alguns métodos, particularmente as abordagens morfológicas; 3) a dificuldade de descrever formas complexas.

Numa tentativa de superar estas dificuldades desenvolveu-se a aplicação CraMs [2]-[5]. Até agora, esta aplicação interativa facilitava a recolha de medidas por parte dos antropólogos, reduzindo o erro na análise e ultrapassando a questão do manuseamento dos espécimes.

Ultrapassadas as questões relativas à análise métrica, incide-se agora na abordagem morfológica. Alguns dos métodos frequentemente utilizados para a estimativa da ancestralidade, particularmente em casos forenses, baseiam-se numa análise qualitativa de características cranianas, especificamente da zona da face [1]. Porém, esta abordagem é, por vezes, criticada por ser subjetiva e fortemente influenciada pela experiência do perito [1], [5]. Com o objetivo de reduzir a subjetividade, Hefner [6] recomendou a observação de imagens desenvolvidas pelo Osteoware (programa desenvolvido para auxiliar na documentação de restos de esqueletos humanos [7]) que permite a avaliação de onze características morfológicas que o autor verificou serem as mais discriminantes na estimativa da ancestralidade. Sendo assim o primeiro programa que dá assistência na classificação das características morfológicas através de descrições detalhadas, e apresentação de dezenas de ilustrações.

Este artigo apresenta uma breve descrição da aplicação CraMs. De seguida são apresentados os novos métodos desenvolvidos para análise de características morfológicas permitindo assim a classificação da ancestralidade do individuo. Também são apresentados os resultados obtidos a partir de uma amostra de cinquenta crânios de ancestralidades europeia e africana.

\section{A APLICAÇÃO CRAMS E MODELOS 3D}

A aplicação CraMs (Fig. 1) permite o alinhamento de crânios, a deteção e marcação de pontos craniométricos e de estruturas (e.g. órbitas e suturas). Posteriormente, estes pontos são usados no cálculo das medidas craniométricas utilizadas nas estimativas do sexo e da ancestralidade [2], [5]. A aplicação foi desenvolvida usando $\mathrm{C}++$, beneficiando das inúmeras bibliotecas disponíveis, tais como VTK (Toolkit de visualização 3D, processamento de imagem e interação)[8] e QT (framework de aplicações multiplataforma que fornece ferramentas para a construção da interface de utilizador) [2], [3], [9]. Mais informações acerca da aplicação CraMs podem ser encontradas em [2]-[5].

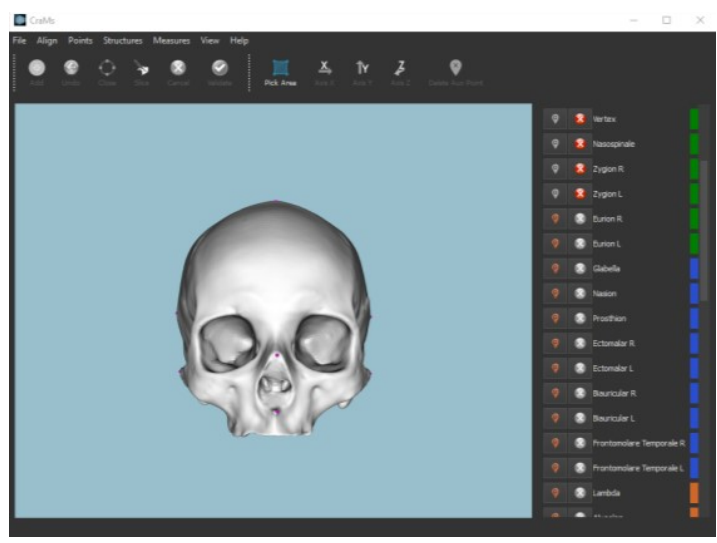

Figura 1 - Aspeto da aplicação CraMs. 
Os modelos 3D usados neste trabalho, um recurso fundamental da aplicação, foram adquiridos usando o scanner NextEngine $^{\mathrm{TM}}$. Este scanner tem um custo relativamente reduzido, é de fácil utilização, tem uma precisão de $125 \mu \mathrm{m}$ e dá origem a modelos com cerca de 0,4 milhões de pontos.

\section{CARÁCTERES NÃO MÉTRICOS}

O método desenvolvido por Hefner não elimina por completo a subjetividade na classificação das características morfológicas, por isso a sua automatização seria um importante passo nessa direção. Nesta secção vamos referir as características morfológicas definidas por Hefner, explicar quais os métodos desenvolvidos para automatizar a sua deteção e classificação, assim como as razões pelas quais algumas características não foram abordadas.

Para estimar a ascendência de um indivíduo, podemos recorrer a métodos métricos e morfológicos desenvolvidos para o esqueleto craniano e pós-craniano. O estudo do crânio pose ser feito baseado em pontos craniométricos ou na análise de características morfológicas. $\mathrm{O}$ crânio é a região do esqueleto onde podemos encontrar o maior número de características, permitindo a análise de afinidades populacionais [10]-[13]. Muitas das características cranianas permitem a diferenciação entre dois dos principais grupos ancestrais, mas não conseguem distinguir todas eles, o que levou à criação de extensas listas de características para avaliações [6], [14]. Hefner [6] descobriu novos traços e o desenvolvimento de novas combinações de traços de modo a acrescentar mais valor a este método. Este autor identificou onze características morfológicas que permitem estimar a ancestralidade em diferentes grupos populacionais (Africanos, Nativos-Americanos, Asiáticos e Europeus): a espinha nasal anterior (ANS), a abertura nasal inferior (INA), a largura interorbital (IOB), o tubérculo malar (MT), a largura da abertura nasal (NAW), o contorno do osso nasal (NBC), o crescimento nasal (NO), a depressão pós-bregmática (PBD), a sutura supranasal (SPS), a sutura palatina transversa (TPS) e a sutura zigomaxilar (ZS). A classificação destas caraterísticas é feita por comparação visual com ilustrações e, portanto, é subjetiva e com resultados difíceis de replicar, sendo assim e como não existe nenhum método ou aplicação desenvolvida que permita detetar e classificar as características definidas por Hefner, um dos objetivos deste trabalho é tentar automatizar a análise de algumas dessas caraterísticas conseguindo assim aumentar a robustez do processo.

Foi dada prioridade à depressão pós-bregmática e às estruturas anatómicas da região nasal, mais especificamente à largura da abertura nasal, à espinha nasal anterior, à abertura nasal inferior e ao contorno do osso nasal. A exclusão da análise dos carateres associados a suturas deveu-se ao facto do método implicar necessariamente uma interação do utilizador, como concluído num estudo prévio [4], em que, com base em dois pontos de referência marcados pelo utilizador, a aplicação encontra um caminho que os liga baseando-se na curvatura. Outra razão é que muitas destas suturas estão ausentes devido a obliteração (por efeito da idade do indivíduo) ou pelo facto da precisão dos modelos 3D não permitir a sua identificação. A região onde se observa o crescimento nasal é composta por peças ósseas muito frágeis e, consequentemente, encontra-se danificada na maioria dos casos, motivo pelo qual este carácter foi excluído. A observação da largura interorbital e do tubérculo malar depende da localização prévia das suturas, tendo sido por isso também excluídas estas características do presente estudo.

\section{A. Largura da abertura nasal}

Definiu-se como objetivo a integração na aplicação CraMs de um método totalmente automático para extrair a largura da abertura nasal e compará-la com a classificação proposta por Hefner [6] (Fig. 2).
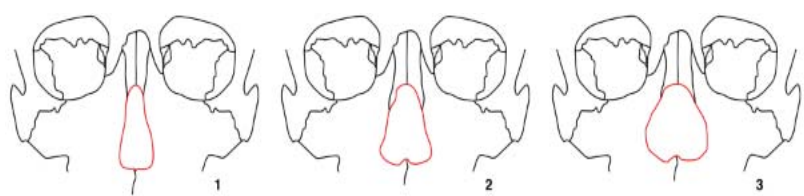

Figura 2 - Os diferentes estados de classificação da largura da abertura nasal (Tipo 1, 2 e 3) (adaptado de Hefner [6]).

\section{Deteção dos pontos craniométricos}

O primeiro passo do processo de deteção da NAW é a determinação automática de pontos de referência, que delimitem a área da abertura nasal sendo usados como sementes para delinear a estrutura em estudo. Foi definido com os antropólogos que esses pontos de referência seriam os pontos centrais superior e inferior da abertura nasal, o rhinion (rhi) e o nasospinale (ns) respetivamente (localizados no plano sagital). Para este método automático definiu-se o nasospinale como sendo o ponto em que o plano sagital mediano intersecta a linha que une os pontos mais baixos nas margens nasais e o rhinion a extremidade inferior da sutura entre os ossos nasais (Fig. 7).

A deteção dos dois pontos de referência é efetuada analisando a curvatura do crânio ao logo de um corte 2D no plano sagital (Fig.3).
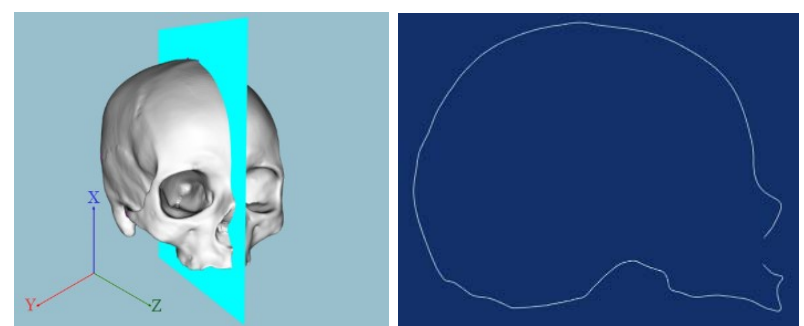

Figura 3 - Plano perpendicular ao plano YX e que intersecta o bregma (esquerda). Secção transversal resultante de intersecção entre o plano e o crânio (direita)

Nesse perfil, o rhinion e o nasospinale correspondem a pontos em que a curvatura muda de direção (pontos críticos) (Fig. 4). Esta condição é combinada com uma análise da vizinhança para evitar falsas deteções devidas a imperfeições do modelo.

Este método permite obter uma lista de pontos críticos que incluem o rhinion e o nasospinale. Contudo, devido ao estado de degradação de alguns crânios, foram adicionadas três novas condições:

- Variações elevadas de curvatura: é aplicado um threshold mínimo de curvatura para validar o ponto. 
- Vizinhança mínima: é considerado apenas o ponto com maior curvatura ao longo de uma dada região.

- Descontinuidade: são assinaladas situações onde a secção transversal a ser analisada não está ligada, onde existem quebras de conectividade.
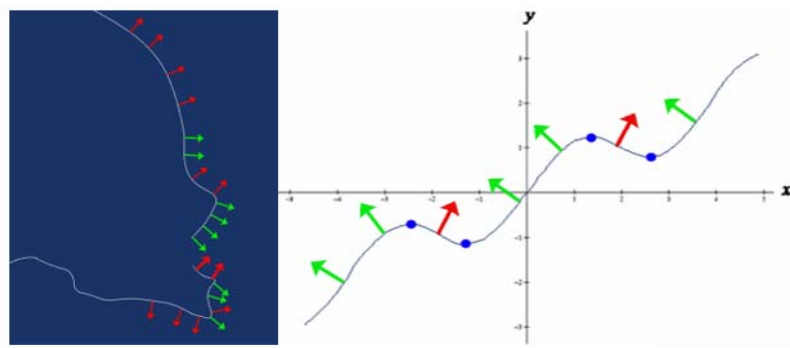

Figura 4 - Representação da normal, com declive positivo (vermelho) e declive negativo (verde) no corte do modelo (esquerda). Representação dos pontos críticos (direita).

Em seguida é necessário filtrar a lista de ocorrências de pontos relevantes (Fig.5), para encontrar as coordenadas apenas dos dois pontos nasais (rhi e ns) que serão usados posteriormente para determinar a NAW.

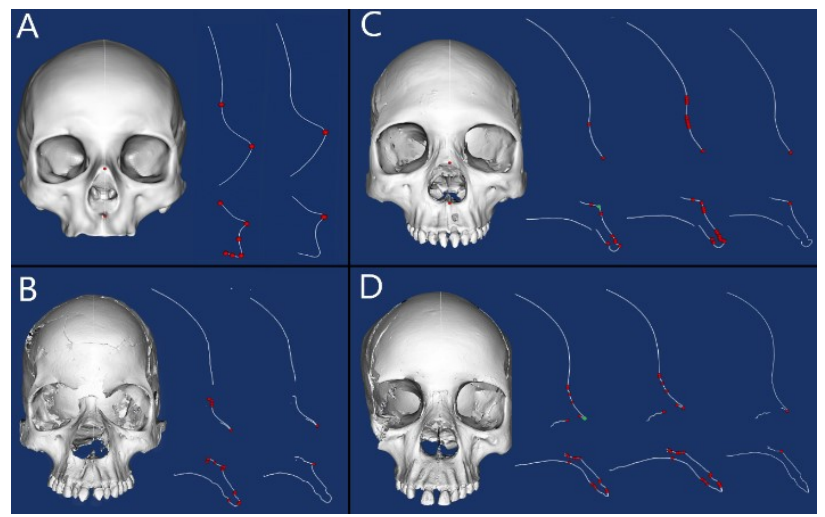

Figura 5 - Crânios com diferentes características e a lista de ocorrências de pontos relevantes.

$\mathrm{O}$ algoritmo começa por determinar o rhinion já que o nasospinale depende da sua localização. O rhinion é facilmente determinado como o ponto com a maior coordenada $\mathrm{Z}$ superior a um threshold definido pelo zygion (zy - ponto lateral mais saliente da arcada zigomática, obtido automaticamente pela aplicação CraMs) (Fig. 6).

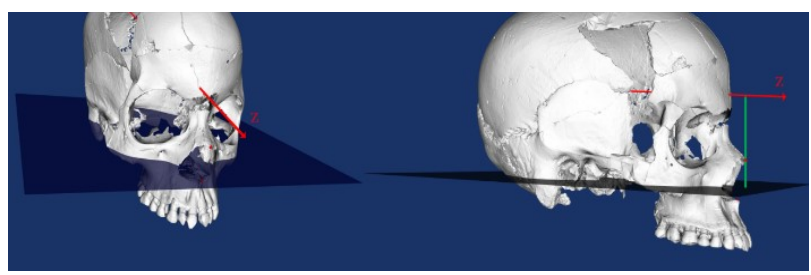

Figura 6 - Plano Zygion - usado para delimitar as áreas de busca do rhinion (localizado acima do plano) e o nasospinale (localizado abaixo do plano).

O nasospinale é aqui definido como o ponto cuja coordenada $\mathrm{Y}$ é inferior à do ponto de zygion e, ao mesmo tempo, tem o valor mais elevado dado pela equação (1), minimiza a distância (d) entre o rhinion e o nasospinale combinando com a ordenada do nasospinale $\left(\mathrm{ns}_{\mathrm{y}}\right)$ de modo a garantir que o ponto não fica no interior da estrutura.

$$
\text { factor }=\frac{n s_{y}}{d}
$$

\section{Deteção de outros pontos semente}

A abertura nasal é a região mais saliente entre o rhinion e o nasospinale. O ponto médio entre esses dois pontos (Fig. 7) é usado como referência para o método que extrai a região nasal. Com base neste ponto, um algoritmo de region growing vai anexando os pontos na vizinhança com propriedades semelhantes. A ideia é determinar zonas de busca através da criação de planos perpendiculares ao plano YX, com ângulos congruentes entre si e com origem no ponto médio (Fig. 7). Procurando os pontos sobre a superfície que têm maior coordenada $\mathrm{Z}$ em cada uma destas regiões, obtém-se o conjunto de pontos semente (tantos quantas as zonas definidas) (Fig. 8).

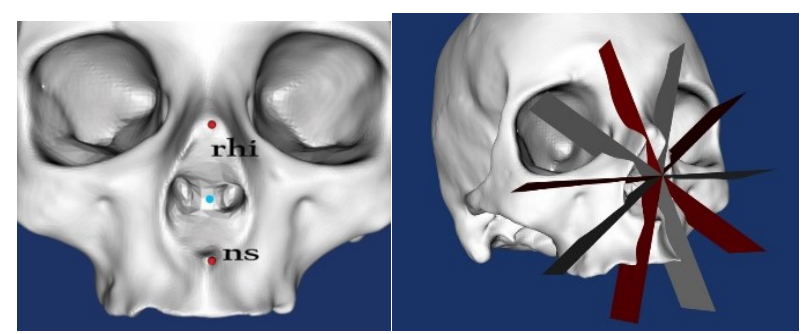

Figura 7 - Localização do nasospinale (ns), do rhinion (rhi) e do ponto médio (esquerda). Ilustração dos planos que limitam as seções de busca (direita).

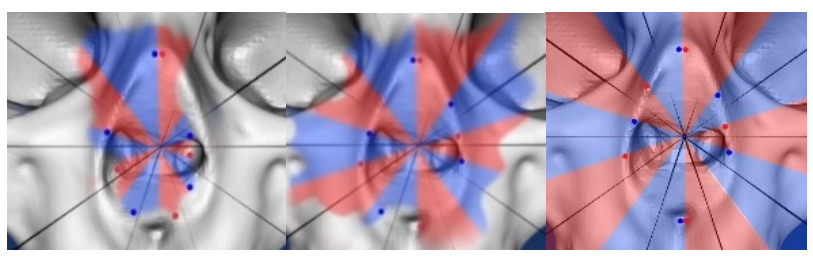

Figura 8 - Pesquisa do ponto com maior coordenada $\mathrm{Z}$ em diferentes seções.

Em testes preliminares, verificou-se que o método falhava em crânios com prognatismo acentuado (projeção anterior do maxilar, característica muito frequente nos indivíduos de ancestralidade africana), que têm uma projeção maior que a estrutura nasal (Fig. 9).

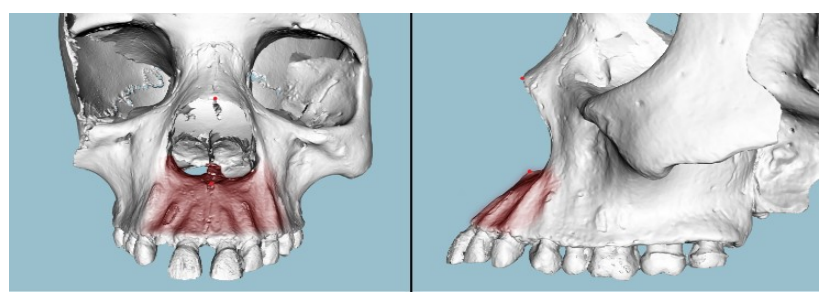

Figura 9-Crânio com maxilar que tem uma projeção mais alargada do que a estrutura nasal (área a vermelho).

Para evitar que fossem detetados pontos fora da cavidade nasal (como se pode observar na Fig. 11 (superior)) foi considerada uma nova condição: os pontos que pertencessem a uma área (Fig. 10) delimitada por dois planos paralelos ao plano YZ (definidos empiricamente pela coordenada $Z$ do $n s \pm 5 \mathrm{~mm}$ ) só seriam considerados se pertencerem à esfera centrada no ponto médio e que passa no nasospinale, evitando assim a seleção de pontos abaixo 
deste. A Fig. 11 mostra os pontos determinados com o método descrito (a verde).

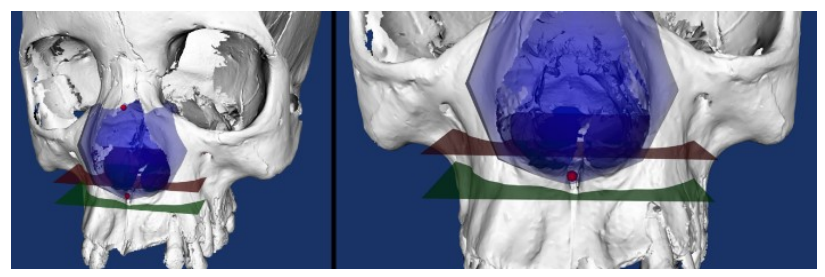

Figura 10 - Área sensível delimitada pelos planos (verde e vermelho) onde os pontos apenas são considerados se estiverem dentro da esfera de referência (azul) centrada no ponto central e passando no nasospinale.

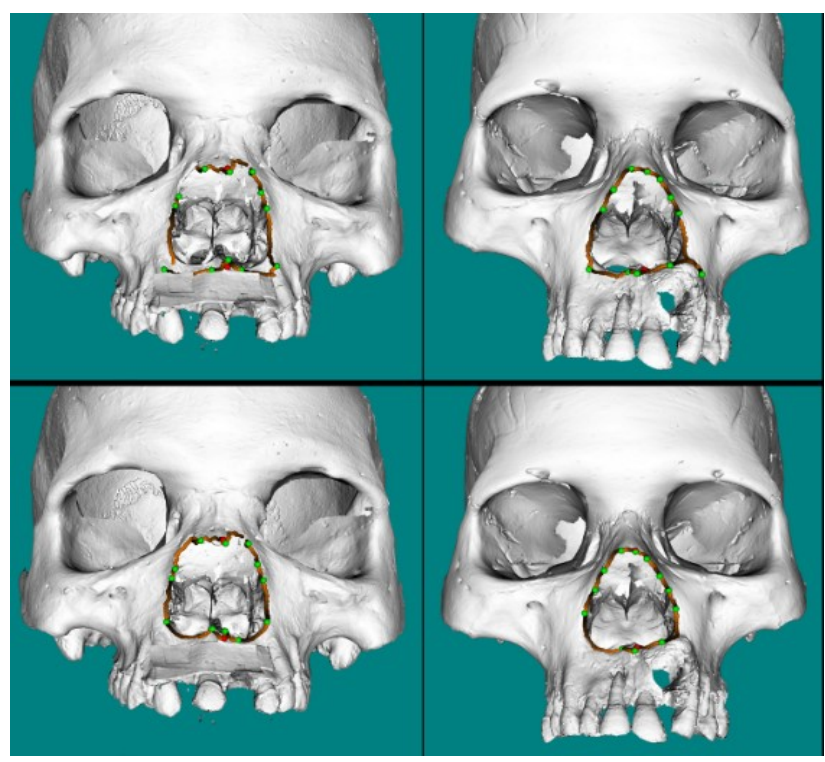

Figura 11 - Exemplos de crânios onde o maxilar tem uma projeção mais alargada do que a estrutura nasal resultando na falha da deteção dos pontos semente nasais inferiores (superior) e resultados com a nova condição (inferior).

\section{Fecho da estrutura nasal}

Depois de alguns testes para avaliar o número adequado de sementes (empiricamente fixado em 12) aplicouse o método de fecho baseado na curvatura descrito em Neves [4]. Este método semiautomático foi usado inicialmente para detetar a estrutura da órbita e fecha um contorno usando pontos semente e analisando a sua vizinhança, com base na curvatura máxima / mínima, de modo a fechar o contorno. Este método foi adaptado para fechar o contorno nasal usando os pontos detetados anteriormente (Fig. 12).

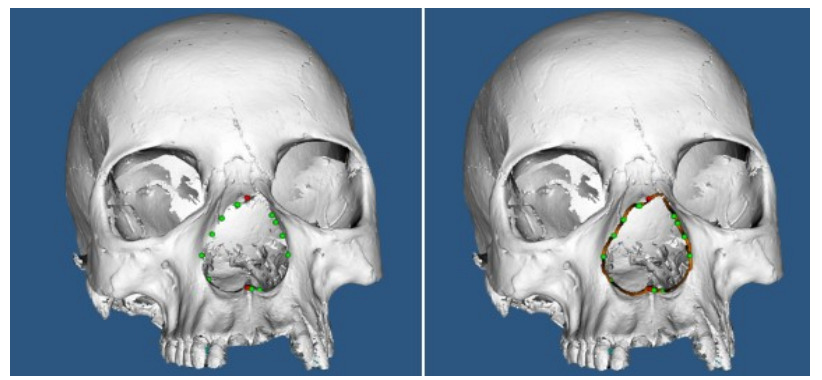

Figura 12 - Fecho do contorno: pontos semente (à esquerda) e resultado do método de análise de curvatura (à direita) usado para unir os pontos semente.

\section{Classificação da estrutura nasal}

O contorno nasal extraído é um polígono 3D. Para permitir a comparação com os perfis 2D de Hefner [6], os pontos do polígono são projetados sobre o plano coronal resultando numa representação 2D da estrutura (Fig. 13).

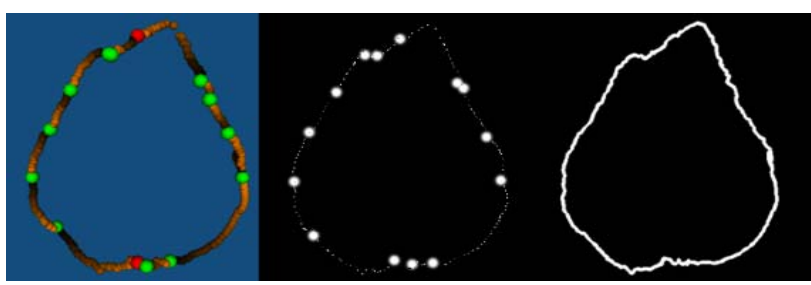

Figura 13 - Imagem 2D obtida a partir da projeção da abertura nasal $3 \mathrm{D}$ sobre o plano coronal.

Para comparar a estrutura 2D assim obtida com as imagens de Hefner [6] (Fig. 15, centro) é necessário assegurar que têm as mesmas proporções, pelo que todas as imagens padrão são centradas e redimensionadas de acordo com a altura da representação 2D da estrutura detetada (Fig.14).

Finalmente são calculadas as distâncias euclidianas entre cada um dos pontos da projeção e o ponto mais próximos de cada perfil original usado por Hefner.
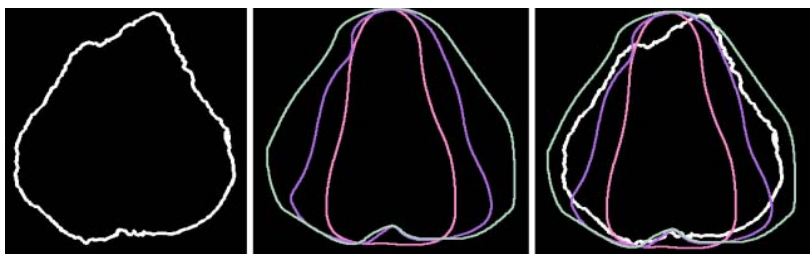

Figura 14 - Representação 2D da estrutura nasal (esquerda), dos 3 perfis de referência definidos por Hefner (centro) e sobreposição de todos os contornos para identificar a correspondência mais próxima (à direita)

Fazendo a média das distâncias entre cada par de imagens, estrutura 2D calculada e a de Hefner, é calculada a probabilidade final de pertencer a uma das classes, definida através da equação (2). Esta equação é obtida através do cálculo de probabilidades aplicado ao espaço amostral resultante do uso da probabilidade inversa (pois quanto menor a distância melhor o resultado).

$$
p_{x}=\frac{d_{1}+d_{2}+d_{3}}{d_{x}^{3} \times \sum_{i=1}^{3} \frac{d_{1}+d_{2}+d_{3}}{d_{i}^{3}}} \times 100
$$

Onde: $p_{\mathrm{x}}$ é a probabilidade de cada uma das classificações e $d_{x}$ é a média da distância euclidiana.

\section{B. Depressão Pós-bregmática}

A depressão pós-bregmática é uma característica morfológica observada no perfil lateral do crânio, que pode ser classificada como presente ou ausente (Fig. 15).

Para determinar este parâmetro é realizada uma projeção do crânio num plano paralelo ao plano sagital, obtendo-se um perfil 2D (ver Fig. 17 esquerda), este processo é baseado nos conceitos de processamento e visualização do VTK [8]. Na segunda fase é obtido o contorno externo desse perfil (ver Fig. 17, direita). 

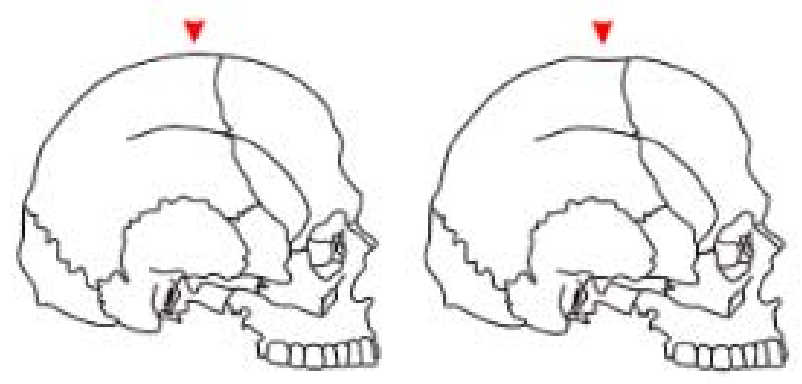

Figura 15 - Os dois estados de classificação da depressão pós-bregmática: ausente (à esquerda) e presente (à direita) (adaptado de Hefner [6]).

Sabendo que a depressão pós-bregmática é definida como uma ligeira depressão ao longo da sutura sagital e posterior ao bregma, são avaliados os pontos entre o bregma (ponto A) e o ponto com maior coordenada Y posterior ao bregma (ponto B) (Fig. 16). A análise consiste em comparar o número de pontos do perfil acima e abaixo de uma linha imaginária entre A e B o que permite determinar a classificação final (Fig. 17).

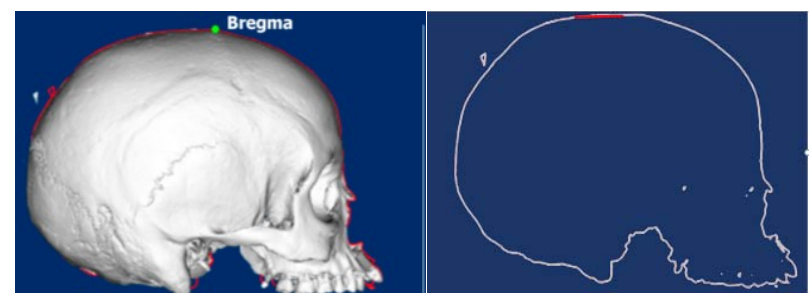

Figura 16 - Projeção lateral (à esquerda) e contorno do perfil do crânio (à direita).
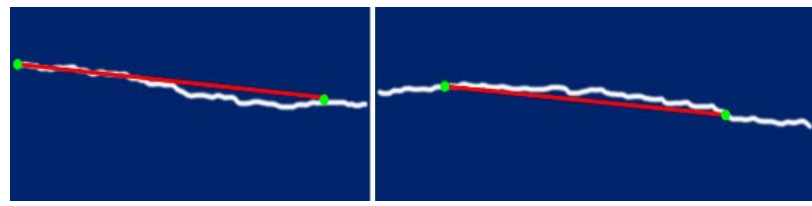

Figura 17 - Depressão pós-bregmática presente (à esquerda) e ausente (à direita).

\section{Espinha Nasal Anterior}

De acordo com Hefner [6], a espinha nasal anterior pode ser classificada como ausente, média ou longa (Fig. 18). Um dos problemas comuns ao classificar a ANS é a fragilidade da área, surgindo muitas vezes danificada. A classificação baseia-se numa análise da projeção da transição do chão nasal para a parte vertical do maxilar, que ocorre na zona do nasospinale.
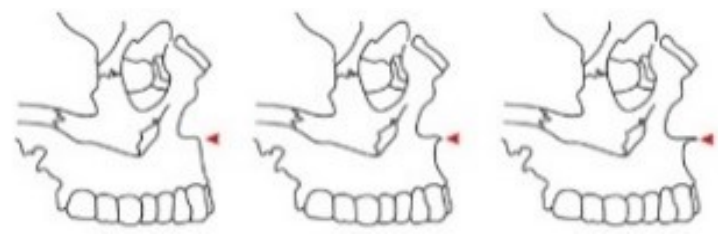

Figura 18 - Diferentes estados de classificação da espinha nasal anterior: ausente (à esquerda), média (centro) e longa (à direita).

Como tal, o método desenvolvido para avaliar esta característica é baseado na posição do nasospinale (detetado pelo processo explicado na secção III.A.1). O próximo passo consiste em analisar os pontos na proximidade do nasospinale e na secção transversal, resultante de um corte feito no plano paralelo a XZ e que passa no nasospinale (Fig.19).

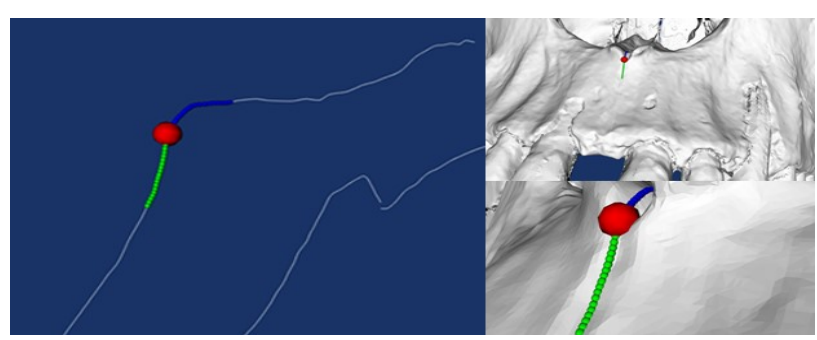

Figura 19 - Crânio (à direita) e secção transversal resultante de um corte feito no plano paralelo ao plano XZ e que passa no nasospinale (à esquerda). O ponto vermelho é o nasospinale; os pontos azuis e verdes são pontos a uma distância inferior a cinco milímetros antes e depois do nasospinale.

Primeiro verifica-se se a projeção da ANS é mais alargada do que a estrutura nasal (Fig. 9), isto é, se todos os pontos têm a coordenada $\mathrm{Z}$ superior à do nasospinale, caso não seja, é classificada como ausente. Isto é possível através da análise dos pontos acima e abaixo do nasospinale (a azul e verde respetivamente na Fig. 20). Caso contrario, para diferenciar uma projeção da ANS média de longa, utilizou-se a equação (3). Se a projeção está fora da área definida pela equação, é designada como média, caso contrário é longa (Fig. 20).

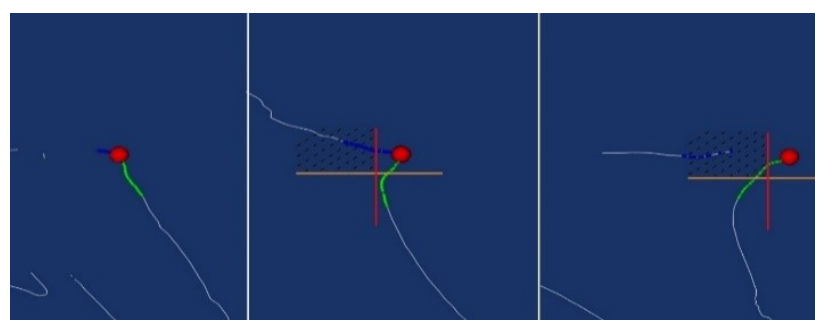

Figura 20 - Classificação da ANS como ausente (à direita), média (ao centro) e longa (à esquerda). A área tracejada permite diferenciar as classificações média e longa.

Os valores que definem a área tiveram de ser ajustados empiricamente, a fim de obter os melhores resultados.

$$
P_{y}>N_{y}-t_{1} \cap P_{z}<N_{z}-t_{1}
$$

Onde: Pz e Py são as coordenadas do ponto que está a ser analisado; Nz e Ny são as coordenadas do ponto a ser analisado; tz e Ty são valores que definem a área.

\section{RESULTADOS E DISCUSSÃO}

Nesta secção são apresentados os resultados obtidos com os métodos anteriormente descritos. Os testes foram feitos em 50 espécimes de duas coleções diferentes, a $\mathrm{CEI} / \mathrm{XXI}$ e a PAVd'.

As classificações foram obtidas usando a aplicação CraMs e através da avaliação de dois dos autores (antropólogos) que classificaram os mesmos espécimes usando o método tradicional, ou seja, recorrendo à comparação com as figuras originais de Hefner [6].

\section{A. Largura da abertura nasal}

Dos cinquenta crânios, 3 estavam demasiado danificados para serem identificados quer manualmente quer 
usando o método descrito, não tendo sido usados neste estudo. Relativamente aos restantes, observou-se uma taxa global de sucesso de $85 \%$ na identificação da largura da abertura nasal. No entanto, em 9\% dos casos os pontos rhinion e em $4 \%$ os pontos nasospinale foram marcados manualmente. Obteve-se uma taxa de sucesso de 95\% na identificação de estruturas do tipo 3, 100\% na identificação de estrutura do tipo 2 e $71 \%$ nas estruturas de tipo 1 (Tabela 1).

Tabela 1 - Resultados das classificações efetuadas segundo o método tradicional (Manual) e o método desenvolvido (Auto). E dada também a precisão probabilística da classificação, isto é, a percentagem da respetiva classificação estar correta para cada um dos três tipos, usando a equação (2).

\begin{tabular}{|c|c|c|c|c|c|}
\hline$I D$ & $\begin{array}{c}\text { NAW } \\
\text { Manual }\end{array}$ & $\begin{array}{l}\text { NAW } \\
\text { Auto }\end{array}$ & & $\%$ & \\
\hline & & & 1 & $\underline{2}$ & $\underline{\mathbf{3}}$ \\
\hline PAVd'_020 & 3 & 3 & $\overline{1}$ & $\overline{5}$ & $\overline{94}$ \\
\hline PAVd'_021 & 3 & 3 & 2 & 30 & 68 \\
\hline PAVd'_025 & 3 & 3 & 2 & 6 & 92 \\
\hline PAVd'_038 & 2 & 2 & 4 & 84 & 12 \\
\hline PAVd'_039 & 3 & 3 & 0 & 3 & 97 \\
\hline PAVd'_041 & 3 & 3 & 0 & 3 & 97 \\
\hline PAVd'_057 & 3 & 3 & 1 & 11 & 88 \\
\hline PAVd'_063 & 2 & 2 & 5 & 92 & 3 \\
\hline PAVd'_065 & 3 & 3 & 3 & 10 & 87 \\
\hline PAVd'_066 & 3 & 3 & 0 & 2 & 98 \\
\hline PAVd'_067 & 3 & 3 & 0 & 1 & 99 \\
\hline PAVd'_069 & 3 & 3 & 0 & 1 & 99 \\
\hline PAVd'_075 & 3 & 3 & 2 & 15 & 83 \\
\hline PAVd'_077 & 3 & 3 & 3 & 25 & 72 \\
\hline PAVd'_078 & 3 & 3 & 9 & 22 & 69 \\
\hline PAVd'_081 & 3 & 3 & 0 & 1 & 99 \\
\hline PAVd'_082 & 3 & 3 & 0 & 0 & 100 \\
\hline PAVd'_093 & 3 & 3 & 1 & 5 & 94 \\
\hline PAVd'_095 & 3 & 3 & 1 & 4 & 95 \\
\hline PAVd'_110 & 3 & 3 & 3 & 28 & 69 \\
\hline PAVd'_133 & 3 & 3 & 0 & 12 & 88 \\
\hline PAVd'_163 & 3 & 2 & 1 & 99 & 0 \\
\hline PAVd'_169 & 3 & 3 & 0 & 5 & 95 \\
\hline CEIXXI_002 & 1 & 1 & 11 & 83 & 6 \\
\hline CEIXXI_003 & 1 & 1 & 72 & 26 & 2 \\
\hline CEIXXI_005 & 1 & 1 & 82 & 17 & 1 \\
\hline CEIXXI_006 & 1 & 2 & 30 & 66 & 4 \\
\hline CEIXXI_009 & 1 & 2 & 11 & 88 & 1 \\
\hline \multicolumn{6}{|l|}{$\begin{array}{l}\text { CEIXXI_011 } \\
\text { CEIXXI_013 }\end{array}$} \\
\hline CEIXXI_014 & 2 & 2 & 29 & 60 & 11 \\
\hline CEIXXI_017 & 1 & 2 & 7 & 77 & 16 \\
\hline CEIXXI_018 & 1 & 1 & 96 & 3 & 1 \\
\hline CEIXXI_019 & 1 & 1 & 74 & 24 & 2 \\
\hline CEIXXI_023 & 1 & 1 & 55 & 42 & 3 \\
\hline \multicolumn{6}{|l|}{ CEIXXI_024 } \\
\hline CEIXXI_026 & 1 & 1 & 67 & 31 & 2 \\
\hline CEIXXI_027 & 1 & 1 & 78 & 20 & 2 \\
\hline CEIXXI_028 & 1 & 1 & 69 & 28 & 3 \\
\hline CEIXXI_036 & 2 & 2 & 14 & 77 & 9 \\
\hline \multicolumn{6}{|l|}{ CEIXXI_040 } \\
\hline CEIXXI_050 & 1 & 2 & 22 & 75 & 3 \\
\hline CEIXXI_069 & 1 & 2 & 17 & 80 & 3 \\
\hline CEIXXI_071 & 2 & 2 & 22 & 69 & 9 \\
\hline CEIXXI_072 & 1 & 1 & 73 & 25 & 2 \\
\hline CEIXXI_076 & 1 & 2 & 14 & 83 & 3 \\
\hline CEIXXI_079 & $2 / 1$ & 2 & 14 & 80 & 6 \\
\hline CEIXXI_088 & 2 & 2 & 5 & 91 & 4 \\
\hline CEIXXI_092 & 2 & 2 & 6 & 61 & 33 \\
\hline CEIXXI_133 & 1 & 2 & 14 & 84 & 2 \\
\hline CEIXXI_135 & 2 & 2 & 6 & 89 & 5 \\
\hline
\end{tabular}

Note-se que vinte e um modelos foram classificados como sendo do tipo 3, nove do tipo 2 e dezassete do tipo 1. O modelo CEIXXI_079 foi o único em que houve discordância entre os dois antropólogos.

Embora se tenha obtido bastante precisão na identificação da NAW, o estado de preservação de alguns crânios, como regiões fragmentadas, provocou um aumento no tempo de execução ou uma identificação incorreta dos dois pontos craniométricos e /ou na classificação da estrutura.

\section{B. Depressão pós-bregmática}

Cinco dos modelos estavam demasiado mal preservados para serem classificados quer manualmente quer usando o método descrito, não sendo possível obter nenhuma informação através da realização de testes nesses modelos. A classificação da depressão pós-bregmática apresentou concordância em $100 \%$ dos casos em que pôde ser avaliada pelo antropólogo. Embora o método desenvolvido para classificar a depressão pós-bregmática apresente resultados promissores, mais modelos com esta característica devem ser adicionados ao estudo, a fim de garantir a eficiência do método, pois esta característica estava presente apenas em três espécimes.

\section{Espinha Nasal Anterior}

Nove dos espécimes não foram passíveis de serem classificados, quer manualmente quer usando o método descrito, por se encontrarem fragmentados. Dez dos modelos eram do tipo 1, dezanove do tipo 2 e doze do tipo 3 . Os resultados da classificação da ANS usando o método desenvolvido, em comparação com a classificação feita pelo antropólogo, apresentou uma taxa de sucesso de $93 \%$ na identificação desta estrutura. Todas as classificações erradas resultaram de incorreta diferenciação entre os tipos 2 e 3 , que pode ser devido a fragmentação de alguns modelos. Para assegurar que os valores escolhidos para selecionar a área que permite uma diferenciação entre o tipo 2 e o tipo 3 estão corretos, são necessários mais modelos destes tipos. Além disso, o método é muito sensível à posição do nasospinale; pequenos erros podem resultar em classificações diferentes.

\section{CONCLUSÕES E PERSPETIVAS FUTURAS}

Neste artigo apresentam-se métodos desenvolvidos para classificação automática de três características morfológicas com o objetivo de ajudar a estimar a ancestralidade dos indivíduos com recurso à aplicação CraMs. Estes métodos foram testados com 50 modelos 3D de crânios de diferentes ancestralidades (europeia e africana) e obtiveram resultados francamente promissores. Embora os métodos ainda requeiram validação com mais crânios (de diferentes origens populacionais) para assegurar os métodos de classificação, os resultados sugerem que este tipo de análise é viável e permite obter resultados análogos aos obtidos através de uma análise tradicional, mas automatizando o processo, tornando-o mais fácil e potencialmente aumentado a sua repetibilidade. Note-se que estes resultados reforçam o potencial da aplicação CraMs sendo a primeira aplicação com deteção e classificação automática 
destas características, bem como da abordagem usada, no campo da Antropologia.

O passo seguinte será desenvolver novos métodos para classificar outras estruturas morfológicas que permitam, por exemplo, estimar o sexo do indivíduo, passo essencial em qualquer análise antropológica. Uma vez que alguns dos espécimes estão fragmentados, um método para ajudar a alinhar os fragmentos e reconstruir a malha nas áreas em falta seria também de grande utilidade.

\section{AGRADECIMENTOS}

Este trabalho foi financiado por fundos nacionais através da FCT - Fundação para a Ciência e Tecnologia, no âmbito do UID projecto / CEC / 00127/2013.

O co-autor David Navega foi financiado pela FCT Fundação para a Ciência e Tecnologia (SFRH/BD/99676/2014). A co-autora Sofia N. Wasterlain foi financiada por fundos nacionais através da Fundação para a Ciência e Tecnologia, no âmbito do projecto estratégico do Cias (FCT-Pest-OE/SADG/UI0283/2013). A co-autora Maria Teresa Ferreira foi financiada pela Gerda Henkel Foundation (AZ 09/F/15).

\section{REFERÊNCIAS}

[1] J. T. Hefner and E. A. DiGangi, "Ancestry estimation," in In Research methods in human skeletal biology, Academic Press, 2013, pp. 117-149.

[2] D. A. Santos, "3D skull models: a new craniometric approach Master Dissertation,” Universidade de Aveiro, 2013.
[3] P. Dias et al., "CraMs, an application for craniometric analysis using 3D skull models," Proc. 17th Int. Conf. In-formation Vis., vol. Jul, no. 1, pp. 462-467, 2013.

[4] L. Neves, "Measures and detection of morphology for craniometry using 3D models. Master Dissertation," Universidade de Aveiro, 2014

[5] P. Dias et al., "CraMs, an application for craniometric analysis using 3D skull models," IEEE Comput. Graph. Appl., vol. 6, no. Nov.-Dec, pp. 11-17, 2015.

[6] J. T. Hefner, "Cranial Nonmetric Variation and Estimating Ancestry," vol. 54, no. 5, pp. 985-995, 2009.

[7] "osteoware." [Online]. Available: https://osteoware.si.edu.

[8] W. Schroeder, K. Martin, and B. Lorensen, Visualization Toolkit: An Object-Oriented Approach to 3D Graphics, 4th Edition, 4th ed. Kitware Inc, 2006.

[9] J. Blanchette, M. Summerfield, M. Gorman, and N. Mcfarlane, $C++$ GUI Programming with Ot 3, vol. 75, no. 9. Hall, Publisher Prentice, 2004.

[10] A. Brues, "The once and future diagnosis of race," in Gill, G. W.; Rhine, S. Skeletal attribution of race: methods for forensic anthropology, Maxwell Museum of Anthropology, University of New Mexico, 1990, pp. 1-7.

[11] S. N. Byers, Introduction to Forensic Anthropology, 4th ed. Pearson, 2011.

[12] G. W. Gill, "Craniofacial Criteria in the Skeletal Attribution of Race," in Reichs, K.J. Forensic osteology: advances in the identification of human remains, 2nd ed., Illinois: Charles C. Thomas Publisher, 1998, pp. 293-315.

[13] L. L. Klepinger, Fundamentals of Forensic Anthropology, 1st ed., no. 1. 2006.

[14] S. Rhine, "Skeletal Criteria for Racial Attribution," NAPA Bull., vol. 13, no. 1, pp. 54-67, Jan. 2008. 